\title{
THE STRONG OKA'S LEMMA, BOUNDED PLURISUBHARMONIC FUNCTIONS AND THE $\bar{\partial}$-NEUMANN PROBLEM*
}

\author{
PHILLIP S. HARRINGTON ${ }^{\dagger}$ AND MEI-CHI SHAW ${ }^{\ddagger}$
}

Dedicated to Salah Baouendi

Key words. Plurisubharmonic function, Oka's lemma, $\bar{\partial}$-Neumann operator

AMS subject classifications. 32F20, 35N15

The classical Oka's Lemma states that if $\Omega$ is a pseudoconvex domain in $\mathbb{C}^{n}$, $n \geq 2$, then $-\log \delta$ is plurisubharmonic where $\delta$ is some distance function to the boundary. Let $M$ be a complex hermitian manifold with the metric form $\omega$. Let $\Omega$ be relatively compact pseudoconvex domain in $M$. We say that a distance function $\delta$ to the boundary $b \Omega$ satisfies the strong Oka condition if it can be extended from a neighborhood of $b \Omega$ to $\Omega$ such that $\delta$ satisfies

$$
i \partial \bar{\partial}(-\log \delta) \geq c_{0} \omega \quad \text { in } \Omega
$$

for some constant $c_{0}>0$.

In this note we first study the relation between the strong Oka's Lemma and the existence of bounded strictly plurisubharmonic functions on a pseudoconvex domain in a complex manifold with $C^{2}$ boundary. The existence of Hölder continuous bounded plurisubharmonic exhaustion functions for pseudoconvex domains with $C^{2}$ boundary in $\mathbb{C}^{n}$ or a Stein manifold is proved by Diederich-Fornaess $[\mathrm{DF}]$. A similar result for domains in $\mathbb{C} P^{n}$ is proved in Ohsawa-Sibony [OS]. In this paper we will give a more unified approach to the existence of bounded plurisubharmonic functions using the strong Oka's lemma. We also show the existence and regularity of the $\bar{\partial}$-Neumann operator for pseudoconvex domains in complex Kähler manifolds with nonnegative curvature, extending earlier results of $[\mathrm{BC}]$ and $[\mathrm{CSW}]$. There are also many other applications of bounded plurisubharmonic functions (see $[\mathrm{DF}],[\mathrm{GW}]$ and $[\mathrm{De}]$ ).

A quantitative approach to the strong Oka's Lemma with compactness and subellipticity for the $\bar{\partial}$-Neumann operator has been obtained recently in [Ha] for pseudoconvex domains in $\mathbb{C}^{n}$. In particular, if (0.1) is satisfied for some positive continuous function $c_{0}=c_{0}(z)$ for $z \in \Omega$ and $c_{0}(z) \rightarrow \infty$ as $z \rightarrow b \Omega$, then the $\bar{\partial}$-Neumann operator is compact. Furthermore, the $\bar{\partial}$-Neumann operator is subelliptic if $c_{0}$ in $(0.1)$ goes to infinity by some inverse fractional order of the distance function. Such results hold for a pseudoconvex domain with Lipschitz boundary in $\mathbb{C}^{n}$. This gives another description of the finite type condition for pseudoconvex domains (see Kohn [Ko1], D'Angelo [DA] and Catlin [Ca]). In Section 3 we give a more streamlined proof of this result when the domain is $C^{2}$ pseudoconvex in a complex Kähler manifold with nonnegative curvature. All our results are stated for domains with $C^{2}$ boundary. It

\footnotetext{
* Received September 1, 2006; accepted for publication March 13, 2007.

† Department of Mathematical Sciences, University of South Dakota, 414 East Clark Street, Vermillion, SD 57069, USA (pharring@usd.edu).

$\ddagger$ Department of Mathematics, University of Notre Dame, Notre Dame, IN 46556, USA (shaw.1@ nd.edu). Partially supported by NSF grants.
} 
is still an open question if one can relax the smoothness of the domain to only $C^{1}$ or Lipschitz boundary in this paper (see the remarks at the end of the paper).

1. The Strong Oka's Lemma and bounded plurisubharmonic exhaustion functions. For a bounded pseudoconvex domain $\Omega$ with $C^{2}$ boundary in $\mathbb{C}^{n}$ or in a Stein manifold, a well known result by Diederich-Fornaess $[\mathrm{DF}]$ shows that there exists a Hölder continuous strictly plurisubharmonic exhaustion function with Hölder exponent $0<\eta<1$. We first examine some equivalent conditions for the existence of such bounded plurisubharmonic functions based on the following simple observation (see [CSW], [CS2]).

Lemma 1.1. Let $M$ be a complex hermitian manifold with metric $\omega$ and let $\Omega \subset \subset$ $M$ be a pseudoconvex domain with $C^{2}$ boundary. Suppose that the distance function $\delta$ satisfies the strong Oka condition (0.1). Then for $0<t_{0} \leq 1$ the following three conditions are equivalent:

(1)

$$
i \partial \bar{\partial}(\log \delta) \geq i t_{0} \frac{\partial \delta \wedge \bar{\partial} \delta}{\delta^{2}}
$$

$$
i \partial \bar{\partial}\left(-\delta^{t_{0}}\right) \geq 0 .
$$

(3) For any $0<t<t_{0}$, there exists some constant $C_{t}>0$ such that

$$
i \partial \bar{\partial}\left(-\delta^{t}\right) \geq C_{t} \delta^{t}\left(\omega+i \frac{\partial \delta \wedge \bar{\partial} \delta}{\delta^{2}}\right) .
$$

In particular, $-\delta^{t}$ is a Hölder continuous strictly plurisubharmonic exhaustion function for $\Omega$.

Proof. If (1.1) holds, we have

$$
i \partial \bar{\partial}(-\log \delta)=i \frac{\partial \bar{\partial}(-\delta)}{\delta}+\frac{i \partial \delta \wedge \bar{\partial} \delta}{\delta^{2}} \geq i t_{0} \frac{\partial \delta \wedge \bar{\partial} \delta}{\delta^{2}} .
$$

Condition (2) is equivalent to

$$
i \frac{\partial \bar{\partial}(-\delta)}{\delta}+\left(1-t_{0}\right) \frac{i \partial \delta \wedge \bar{\partial} \delta}{\delta^{2}} \geq 0 .
$$

Comparing this with (1.4), it is easy to see that (1) and (2) are equivalent (without any assumption on the strong Oka condition for $\delta$ ).

Assume that the distance function $\delta$ satisfies the strong Oka condition (0.1). To see that (2) and (3) are equivalent, we multiply (1.4) by $(1-\epsilon)$ and (0.1) by $\epsilon$. Adding the two inequalities, we conclude that, for any $0 \leq \epsilon \leq 1$, the inequality

$$
i \partial \bar{\partial}(-\log \delta)=i \frac{\partial \bar{\partial}(-\delta)}{\delta}+\frac{i \partial \delta \wedge \bar{\partial} \delta}{\delta^{2}} \geq c_{0} \epsilon \omega+(1-\epsilon) t_{0} \frac{i \partial \delta \wedge \bar{\partial} \delta}{\delta^{2}}
$$


holds. Hence, for any $0<t<t_{0}$, we choose $\epsilon=\epsilon_{t}$ such that $\left(1-\epsilon_{t}\right) t_{0}>t$. Then

$$
\begin{aligned}
i \partial \bar{\partial}\left(-\delta^{t}\right) & =i t \delta^{t}\left(\frac{\partial \bar{\partial}(-\delta)}{\delta}+(1-t) \frac{\partial \delta \wedge \bar{\partial} \delta}{\delta^{2}}\right) \\
& =i t \delta^{t}\left(\partial \bar{\partial}(-\log \delta)-t \frac{\partial \delta \wedge \bar{\partial} \delta}{\delta^{2}}\right) \\
& \geq C_{t} t \delta^{t}\left(\omega+\frac{i \partial \delta \wedge \bar{\partial} \delta}{\delta^{2}}\right)
\end{aligned}
$$

where $C_{t}=\min \left(c_{0} \epsilon_{t},\left(1-\epsilon_{t}\right) t_{0}-t\right)$. This gives that (2) implies (3).

To see that (3) implies (2), we use the assumption that the boundary is $C^{2}$. This implies that that the function $\delta$ is $C^{2}$. Condition (3) implies that $i \partial \bar{\partial}\left(-\delta^{t}\right) \geq 0$ for every $0<t<t_{0}$. Condition (2) follows from (3) by continuity. This proves that (2) and (3) are equivalent. The lemma is proved.

TheOREM 1.2. Let $M$ be a complex hermitian manifold and let $\Omega \subset \subset M$ be a pseudoconvex domain with $C^{2}$ boundary $b \Omega$. Let $\delta(x)=d(x, b \Omega)$ be the distance function to $b \Omega$ with respect to the hermitian metric such that $\delta$ satisfies the strong Oka condition (0.1). Then there exists $0<t_{0} \leq 1$ such that

$$
i \partial \bar{\partial}(-\log \delta) \geq i t_{0} \frac{\partial \delta \wedge \bar{\partial} \delta}{\delta^{2}} .
$$

Proof. Near a boundary point, we choose a special orthonormal basis $w_{1}, \cdots, w_{n}$ for $(1,0)$-forms such that $w_{n}=\sqrt{2} \partial \delta$. Let $L_{1}, \cdots, L_{n}$ be its dual. Let $a$ be any $(1,0)$-vector. We decompose $a=a_{\tau}+a_{\nu}$ where $a_{\nu}=\left\langle a, L_{n}\right\rangle$ is the complex normal component and $a_{\tau}$ is the complex tangential component. We have

$$
\begin{aligned}
& \langle\partial \bar{\partial}(-\log \delta), a \wedge \bar{a}\rangle \\
& =\left\langle\frac{\partial \bar{\partial}(-\delta)}{\delta}, a_{\tau} \wedge \bar{a}_{\tau}\right\rangle+2 \Re\left\langle\frac{\partial \bar{\partial}(-\delta)}{\delta}, a_{\tau} \wedge \bar{a}_{\nu}\right\rangle \\
& +\left\langle\frac{\partial \bar{\partial}(-\delta)}{\delta}, a_{\nu} \wedge \bar{a}_{\nu}\right\rangle+\frac{\left|a_{\nu}\right|^{2}}{\delta^{2}} .
\end{aligned}
$$

From (0.1) and the equality in (1.4), we have

$$
\left\langle\frac{\partial \bar{\partial}(-\delta)}{\delta}, a_{\tau} \wedge \bar{a}_{\tau}\right\rangle \geq c_{0}\left|a_{\tau}\right|^{2} .
$$

If $\delta$ is $C^{2}$ up to the boundary, we have for any $\epsilon>0$,

$$
\left|\left\langle\frac{\partial \bar{\partial}(-\delta)}{\delta}, a_{\tau} \wedge \bar{a}_{\nu}\right\rangle\right| \leq C\left(\frac{1}{\epsilon}\left|a_{\tau}\right|^{2}+\epsilon \frac{\left|a_{\nu}\right|^{2}}{\delta^{2}}\right) .
$$

Also near the boundary when $\delta(z)<\epsilon$, we have

$$
\left|\left\langle\frac{\partial \bar{\partial}(-\delta)}{\delta}, a_{\nu} \wedge \bar{a}_{\nu}\right\rangle\right| \leq \frac{C}{\delta}\left|a_{\nu}\right|^{2} \leq C \frac{\epsilon}{\delta^{2}}\left|a_{\nu}\right|^{2} .
$$

Substituting (1.6)-(1.8) into (1.5) and choosing $\epsilon$ sufficiently small, we have

$$
\langle\partial \bar{\partial}(-\log \delta), a \wedge \bar{a}\rangle \geq \frac{1}{2} \frac{\left|a_{\nu}\right|^{2}}{\delta^{2}}-K\left|a_{\tau}\right|^{2}
$$


for some large constant $K$. Multiplying (0.1) by $\frac{K}{c_{0}}$ and adding it to (1.9), we have

$$
\left(\frac{K}{c_{0}}+1\right)\langle\partial \bar{\partial}(-\log \delta), a \wedge \bar{a}\rangle \geq \frac{1}{2} \frac{\left|a_{\nu}\right|^{2}}{\delta^{2}} .
$$

This proves (1.4) with $t_{0}=\frac{1}{2\left(\frac{K}{c_{0}}+1\right)}$ near the boundary. The theorem is proved.

Corollary 1.3. Let $\Omega, \delta(x)$ and $t_{0}$ be the same as in Theorem 1.2. Then for any $0<t<t_{0}$, the function $\tilde{\delta}=-\delta^{t}$ is a strictly plurisubharmonic bounded exhaustion function on $\Omega$.

The corollary follows immediately from the equivalence of (1) and (3) in Lemma 1.1. From Theorem 1.2, we have the following Diederich-Fornaess Theorem [DF].

Theorem 1.4 (Diederich-Fornaess). Let $\Omega \subset \subset M$ be a pseudoconvex domain with $C^{2}$ boundary in a Stein manifold $M$. Then there exists a defining function $\rho$ of class $C^{2}$ and some number $0<t<1$ such that $\tilde{\delta}=-(-\rho)^{t}$ is a strictly plurisubharmonic bounded exhaustion function on $\Omega$.

Proof. Since $M$ is Stein, $M$ can be embedded in $\mathbb{C}^{N}$ for some large $N$. Let $\delta(x)=d(x, b \Omega)$ be the distance function to $b \Omega$ with respect to metric $\omega$ induced by the Euclidean metric in $\mathbb{C}^{N}$. From Oka's Lemma, we have $i \partial \bar{\partial}(-\log \delta) \geq 0$ in a neighborhood $U$ of $b \Omega$.

Let $\phi$ be a smooth strictly plurisubharmonic function on $M$. For any $c_{0}>0$, we can choose some large $\lambda>0$ such that

$$
i \partial \bar{\partial}\left(-\log \left(\delta e^{-\lambda \phi}\right)\right)=-i \partial \bar{\partial} \log \delta+\lambda \omega \geq c_{0} \omega
$$

where $\omega$ is the metric form induced by the Euclidean metric. Thus the strong Oka condition (0.1) holds. The theorem follows from Corollary 1.3.

REMARK. In the proof of Theorem 1.4, if $\Omega$ is in $\mathbb{C}^{n}$, we can choose $\phi=|z|^{2}$.

When the complex manifold is Kähler with positive curvature, we have also the following result of Ohsawa-Sibony [OS].

Theorem 1.5 (Ohsawa-Sibony). Let $\Omega \subset \subset \mathbb{C} P^{n}$ be a pseudoconvex domain with $C^{2}$ boundary $b \Omega$ and let $\delta(x)=d(x, b \Omega)$ be the distance function to $b \Omega$ with the Fubini-Study metric $\omega$. Then there exists $t_{0}=t_{0}(\Omega)$ with $0<t_{0} \leq 1$ such that

$$
i \partial \bar{\partial}\left(-\delta^{t_{0}}\right) \geq 0 \text {. }
$$

This follows easily from Takeuchi's Theorem (see [Ta], also [CS1])

$$
i \partial \bar{\partial}(-\log \delta) \geq c_{0} \omega
$$

where $c_{0}$ can be chosen to be equal to $\frac{1}{2}$.

REMARKs. 1. The theorems above show that if either the complex manifold has positive curvature or there is a positive line bundle, then the strong Oka's lemma holds. Both theorems do not hold without the positivity condition on the metric (see the counterexample in $[\mathrm{DF}]$ and Theorem 1.2 in $[\mathrm{OS}])$. 
2. If $\Omega$ is a Lipschitz bounded pseudoconvex domain in a Stein manifold, it is proved in Demailly [De] that there exists a bounded strictly plurisubharmonic function in $\Omega$ (see also Kerzman-Rosay [KeR] for the $C^{1}$ case). It is not known if this is true for pseudoconvex domains with Lipschitz boundary in $\mathbb{C} P^{n}$. We also remark that strictly plurisubharmonic bounded exhaustion functions might not exist if the Lipschitz boundary (as a graph) condition is dropped (see [DF]).

2. The $\bar{\partial}$-Neumann problem on Kähler manifolds with nonnegative curvature. Let $M$ be a complex manifold with a hermitian metric $\omega$ and let $\Omega \subset \subset M$ be a pseudoconvex domain. If $M$ is Stein, the $L^{2}$ existence theorems for $\bar{\partial}$ and the $\bar{\partial}$-Neumann problem follow from Hörmander's theory [Hö2]. In the case when the manifold $M$ is Kähler with negative curvature, the distance function to a fixed point is a strictly plurisubharmonic function and hence, $M$ is Stein (see [GW]). In this section we study the $L^{2} \bar{\partial}$ theory when the manifold is not Stein and there is no strictly plurisubharmonic weight function smooth up to the boundary. One has to modify Hörmander's weighted method to establish the $L^{2}$ theory for $\bar{\partial}$.

Suppose that the manifold has positive curvature, like $\mathbb{C} P^{n}$. Then there exists some distance function $\delta$ for $\Omega$ which satisfies the strong Oka's condition (0.1). Then we can use $\phi=-\log \delta$ to be the weight function in Hörmander's theory and study the weighted $\bar{\partial}$-Neumann problem (see $[\mathrm{Si}]$ or $[\mathrm{CS}]$ ). However, $\phi$ is not continuous up to the boundary. To establish the $L^{2}$ theory without weights, we use an idea by Berndtsson-Charpentier $[\mathrm{BC}]$ and streamlined in [CSW].

Let $t$ be any real number and $\phi \in C^{2}(\Omega)$. Let $L^{2}\left(\delta^{t}\right)$ denote the $L^{2}$ space with respect to the weight function $e^{-t \phi}=\delta^{t}$ and

$$
\|f\|_{(t)} \equiv\|f\|_{L^{2}\left(\delta^{t}\right)}^{2}=\int_{\Omega}|f|^{2} e^{-t \phi}=\int_{\Omega} \delta^{t}|f|^{2} .
$$

We use $\bar{\partial}_{t}^{*}$ to denote the adjoint of $\bar{\partial}$ with respect to the weighted space. Then $\bar{\partial}_{t}^{*}=\delta^{-t} \vartheta \delta^{t}$ whenever it is defined, where $\vartheta$ denotes the formal adjoint with respect to the unweighted $L^{2}$-norm. The norm $\|\cdot\|_{(t)}^{2}$ is equivalent to the Sobolev norm on a sub-space of $W^{-\frac{t}{2}}(\Omega)$ for harmonic functions or solutions to elliptic equations.

Let $\delta$ be a distance function which satisfies the strong Oka condition (0.1). Introduce the following two asymmetric weighted norms. These new norms will be used to obtain more refined $L^{2}$ estimates.

For any $(p, q)$-form $f$ on $\Omega$, we decompose $f$ into complex normal and tangential parts by setting

$$
\begin{cases}f^{\nu} & =\left(f\left\llcorner(\bar{\partial} \delta)_{\#}\right) \wedge \bar{\partial} \delta\right. \\ f^{\tau} & =f-f^{\nu}\end{cases}
$$

The above decomposition is well-defined for any $(p, q)$-form $f$ supported near the boundary and can be extended to the whole domain.

We define the asymmetric weighted norm

$$
|f|_{A}^{2}=\left|f^{\tau}\right|^{2}+\frac{\left|f^{\nu}\right|^{2}}{|\delta|^{2}}
$$

and its dual norm

$$
|f|_{A^{\prime}}^{2}=\left|f^{\tau}\right|^{2}+\left|f^{\nu}\right|^{2}|\delta|^{2}
$$


For any $t>0$, let $L_{A}^{2}\left(\delta^{t}\right)$ and $L_{A^{\prime}}^{2}\left(\delta^{t}\right)$ denote the weighted $L^{2}$ spaces on $(p, q)$-forms defined by the norm

$$
\|u\|_{L_{A}^{2}\left(\delta^{t}\right)}^{2}=\int_{\Omega} \delta^{t}|f|_{A}^{2}=\int_{\Omega} \delta^{t}\left(\left|f^{\tau}\right|^{2}+\frac{\left|f^{\nu}\right|^{2}}{|\delta|^{2}}\right)
$$

and

$$
\|u\|_{L_{A^{\prime}}^{2}\left(\delta^{t}\right)}^{2}=\int_{\Omega} \delta^{t}|f|_{A^{\prime}}^{2}=\int_{\Omega} \delta^{t}\left(\left|f^{\tau}\right|^{2}+\left|f^{\nu}\right|^{2}|\delta|^{2}\right) .
$$

THEOREM 2.1. Let $M$ be a complex Kähler manifold with nonnegative sectional curvature. Let $\Omega \subset \subset M$ be a pseudoconvex domain with $C^{2}$-smooth boundary $b \Omega$. Let $\delta(x)=d(x, b \Omega)$ be the distance function such that $\delta$ satisfies the strong Oka condition (0.1). For any $f \in L_{A^{\prime}(p, q)}^{2}(\Omega)$, where $0 \leq p \leq n$ and $1 \leq q \leq n$, such that $\bar{\partial} f=0$ in $\Omega$, there exists $u \in L_{(p, q-1)}^{2}(\Omega)$ satisfying $\bar{\partial} u=f$ and

$$
\int_{\Omega}|u|^{2} \leq C \int_{\Omega}|f|_{A^{\prime}}^{2}
$$

Proof. We first show that for any $t>0$ and any $(p, q)$-form $f \in L_{A^{\prime}}^{2}\left(\delta^{t}\right), 0 \leq p \leq n$ and $1 \leq q \leq n$, such that $\bar{\partial} f=0$ in $\Omega$, there exists $u \in L_{(p, q-1)}^{2}\left(\delta^{t}\right)$ satisfying $\bar{\partial} u=f$ and

$$
\|u\|_{L^{2}\left(\delta^{t}\right)}^{2} \leq \frac{C}{t}\|f\|_{L_{A^{\prime}}^{2}\left(\delta^{t}\right)}^{2} .
$$

Let $\phi=-t \log \delta$, where $t>0$. By the Bochner-Hörmander-Kohn-Morrey formula with weight function $\phi=-t \log \delta$, for any $(p, q)$-form $g \in \operatorname{Dom}(\bar{\partial}) \cap \operatorname{Dom}\left(\bar{\partial}^{*}\right)$ with $q \geq 1$ on $\Omega$, we have

$$
\|\bar{\partial} g\|_{\phi}^{2}+\left\|\bar{\partial}_{\phi}^{*} g\right\|_{\phi}^{2}=\|\bar{\nabla} g\|_{\phi}^{2}+(\Theta g, \bar{g})_{\phi}+((i \partial \bar{\partial} \phi) g, \bar{g})_{\phi}+\int_{b \Omega}\langle(i \partial \bar{\partial} \rho) g, \bar{g}\rangle e^{-\phi},
$$

where $\|\bar{\nabla} u\|_{\phi}^{2}=\int_{\Omega} \sum_{j=1}^{n}\left|D_{\bar{L}_{j}} u\right|^{2} e^{-\phi},\left\{L_{1}, \ldots, L_{n}\right\}$ is a local unitary frame of $T^{(1,0)}(\Omega)$ and $\Theta$ is a curvature form. From our assumption, we have $(\Theta u, \bar{u})_{\phi} \geq 0$.

Since $\Omega$ is pseudoconvex, we have that for any $(p, q)$-form $g \in \operatorname{Dom}(\bar{\partial}) \cap \operatorname{Dom}\left(\bar{\partial}_{t}^{*}\right)$,

$$
\|\bar{\partial} g\|_{(t)}^{2}+\left\|\bar{\partial}_{t}^{*} g\right\|_{(t)}^{2} \geq((i \partial \bar{\partial} \phi) g, \bar{g})_{(t)} \geq C t\|g\|_{L_{A}^{2}\left(\delta^{t}\right)} .
$$

Let $(i \partial \bar{\partial} \phi)^{\prime}$ denote the dual norm for $(p, q)$-forms induced by $i \partial \bar{\partial} \phi$. It follows that for any $f \in L_{A^{\prime}}^{2}\left(\delta^{t}\right)$, there exist $u \in L^{2}\left(\delta^{t}\right)$ satisfying $\bar{\partial} u=f$ and

$$
\int_{\Omega}|u|^{2} \delta^{t} \leq \int_{\Omega}|\bar{\partial} u|_{(i \partial \bar{\partial} \phi)^{\prime}}^{2} \delta^{t} \leq \frac{1}{C t} \int_{\Omega}|\bar{\partial} u|_{A^{\prime}}^{2} \delta^{t} .
$$

This proves (2.5). To get rid of $t$, we use an argument used in [BC] (see also [HI] or $[\mathrm{CSW}])$. Let $f \in L_{(p, q)}^{2}(\Omega)$. For any $t>0$, there exists $u \in L_{(p, q-1)}^{2}\left(\delta^{t}\right)$ satisfying $\bar{\partial} u=f$, such that $u$ is perpendicular to $\operatorname{Ker}(\bar{\partial})$ in $L^{2}\left(\delta^{t}\right)$ and $u$ satisfies (2.7).

Consider $v=u \delta^{-t}$. Then $v \perp \operatorname{Ker}(\bar{\partial})$ in $L^{2}\left(\delta^{2 t}\right)$. It follows from (2.7) that the following holds:

$$
\int_{\Omega}|u|^{2}=\int_{\Omega}|v|^{2} \delta^{2 t} \leq \frac{1}{2 C_{0} t} \int_{\Omega}|\bar{\partial} v|_{A^{\prime}}^{2} \delta^{2 t} .
$$

Since

$$
|\bar{\partial} v|_{A^{\prime}}^{2} \delta^{2 t} \leq C\left(|\bar{\partial} u|_{A^{\prime}}^{2}+2 t^{2}|u|^{2}\right),
$$


choosing $t$ sufficiently small and substituting (2.9) into (2.8), one obtains

$$
\int_{\Omega}|u|^{2} \leq C_{t} \int_{\Omega}|\bar{\partial} u|_{A^{\prime}}^{2}
$$

This proves the theorem.

THEOREM 2.2. Let $M$ be a complex Kähler manifold with nonnegative sectional curvature. Let $\Omega \subset \subset M$ be a pseudoconvex domain with $C^{2}$-smooth boundary $b \Omega$. Let $\delta(x)=d(x, b \Omega)$ be the distance function such that $\delta$ satisfies the strong Oka condition (0.1). Then $\square_{(p, q)}$ has closed range and the $\bar{\partial}$-Neumann operator $N_{(p, q)}: L_{(p, q)}^{2}(\Omega) \rightarrow$ $L_{(p, q)}^{2}(\Omega)$ exists for every $p, q$ such that $0 \leq p \leq n, 0 \leq q \leq n$. Moreover, for any $f \in L_{(p, q)}^{2}(\Omega)$, we have

$$
\begin{aligned}
& f=\bar{\partial} \bar{\partial}^{*} N_{(p, q)} f \oplus \bar{\partial}^{*} \bar{\partial} N_{(p, q)} f, \quad 1 \leq q \leq n-1 . \\
& f=\bar{\partial}^{*} \bar{\partial} N_{(p, 0)} f \oplus P f, \quad q=0,
\end{aligned}
$$

where $P$ is the orthogonal projection from $L_{(p, 0)}^{2}(\Omega)$ onto $L_{(p, 0)}^{2}(\Omega) \cap \operatorname{Ker}(\bar{\partial})$ and

$$
N_{(p, 0)}=\bar{\partial}^{*} N_{(p, 1)}^{2} \bar{\partial} \text {. }
$$

Furthermore, there exists $0<t_{0} \leq 1$ such that the $\bar{\partial}$-Neumann operator $N, \bar{\partial} N, \bar{\partial}^{*} N$ and the Bergman projection $P$ are exactly regular on $W_{(p, q)}^{s}(\Omega)$ for $0 \leq s<\frac{1}{2} t_{0}$ with respect to the $W^{s}(\Omega)$-Sobolev norms.

The $L^{2}$-existence theorem for the $\bar{\partial}$-Neumann operator $N$ on $\Omega$ follows from the $L^{2}$-existence of the solution $u$ for the $\bar{\partial}$-equation proved in Theorem 2.1.

To show that $N$ is regular in $W^{s}$ for $s<\frac{1}{2} t_{0}$, let $t=2 s$ in the Bochner-HörmanderKohn-Morrey formula with weight function $\phi=-t \log \delta$. After rearranging terms, we have for any $(p, q)$-form $g \in \operatorname{Dom}(\bar{\partial}) \cap \operatorname{Dom}\left(\bar{\partial}^{*}\right)$,

$$
\begin{aligned}
\|\bar{\partial} g\|_{(t)}^{2} & +\left\|\bar{\partial}^{*} g\right\|_{(t)}^{2}-2 \Re\left(\bar{\partial}^{*} g, \overline{g\left\llcorner\left(\bar{\partial} \delta^{t}\right)_{\sharp}\right.}\right) \\
& =\|\bar{\nabla} g\|_{(t)}^{2}+(\Theta g, \bar{g})_{(t)}-\left(\left(i \partial \bar{\partial}\left(\delta^{t}\right) g, \bar{g}\right) .\right.
\end{aligned}
$$

(see Proposition 3.1 in [CSW]). Since for any $\epsilon>0$ we have

$$
\left|2 \Re\left(\bar{\partial}^{*} g, \overline{g\left\llcorner\left(\bar{\partial} \delta^{t}\right)_{\sharp}\right.}\right)\right| \leq \frac{1}{\epsilon}\left\|\bar{\partial}^{*} g\right\|_{(t)}^{2}+\epsilon t^{2}\left\|\frac{g^{\nu}}{\delta}\right\|_{(t)}^{2},
$$

choosing $\epsilon$ small, we have from (2.11), (2.12) and (1.3) that

$$
\begin{aligned}
\|\bar{\partial} g\|_{(t)}^{2}+\left\|\bar{\partial}^{*} g\right\|_{(t)}^{2} & \geq C_{t}\left(\|g\|_{(t)}^{2}+\left\|\frac{g^{\nu}}{\delta}\right\|_{(t)}^{2}\right) \\
& \geq C_{t}\|g\|_{(t)}^{2} .
\end{aligned}
$$

The rest of the proof is similar to the proof of Theorem 2 in [CSW], and we omit the details. When $\Omega \subset \subset \mathbb{C}^{n}$, the Sobolev regularity for $\bar{\partial}^{*} N$ and the Bergman projection have been obtained earlier in $[\mathrm{BC}]$ (see also [Ko2]).

Remarks. 1. If $t_{0}=1$ in Theorem 1.2, the domain $\Omega$ has a plurisubharmonic defining function. It follows from Boas-Straube [BS] that the $\bar{\partial}$-Neumann operator is bounded in the Sobolev space $W^{s}$ for all $s>0$ if $\Omega$ has smooth boundary. We also mention that for any $\beta>0$, there exists a smooth bounded pseudoconvex domain $\Omega_{\beta}$ 
in $\mathbb{C}^{n}$ such that the $\bar{\partial}$-Neumann operator is not bounded on $W^{s}$ (see the paper by Barrett [Ba]). It is not known if one can find a $C^{\infty}(\bar{\Omega})$ solution $u$ satisfying $\bar{\partial} u=f$ for $f \in C_{(0,1)}^{\infty}(\bar{\Omega})$ with $\bar{\partial} f=0$ in $\Omega$. In fact, one obtains from Theorem 2.2 only a $W^{s}(\Omega)$ solution for some $s>0$.

2. It is still not known if Theorems 2.1 or 2.2 hold for pseudoconvex domains with $C^{1}$ or Lipschitz boundary.

3. The Strong Oka Condition and Compactness of the $\bar{\partial}$-Neumann Operator. In this section, we consider the possibility that there exists a defining function for $\Omega$ satisfying (0.1) in a stronger sense. More precisely, suppose that there exists some defining function $\rho$ for $\Omega$ satisfying:

$$
i \partial \bar{\partial}(-\log (-\rho)) \geq \phi(-\rho) \omega
$$

in the sense of currents for some continuous function $\phi$ such that $\lim _{x \rightarrow 0^{+}} \phi(x)=+\infty$. It is necessary to work with currents for such functions, since $\rho$ satisfying (3.1) may not be $C^{2}$, even on smooth domains. If this is true on a $C^{2}$ domain, we will show that the $\bar{\partial}$-Neumann operator is compact in $W_{(p, q)}^{s}(\Omega)$ for all $0 \leq s<\frac{1}{2}$. Furthermore, if we can take $\phi(x)=x^{-2 \epsilon}$, then the $\bar{\partial}$-Neumann operator will have a subelliptic gain of $2 \epsilon$. First, we will need a stronger (local) form of Theorem 1.2.

Lemma 3.1. Let $M$ be a complex hermitian manifold and let $\Omega \subset \subset M$ be a pseudoconvex domain with $C^{2}$ boundary $b \Omega$. Let $\rho$ be a defining function for $\Omega$ satisfying (3.1) in $\Omega$. Then for any $0<t<1$ and $p \in b \Omega$, there exists a local defining function $\rho_{p}$ in a neighborhood $U$ of $p$ such that $\rho_{p}$ satisfies:

$$
i \partial \bar{\partial}\left(-\left(-\rho_{p}\right)^{t}\right) \geq C\left(-\rho_{p}\right)^{t} \phi\left(-\rho_{p}\right) \omega
$$

on $\Omega \cap U$ in the sense of currents for some constant $C>0$.

Proof. In the original proof of Theorem 1.4 in $[\mathrm{DF}]$, it is observed that for any $0<t<1$ and $p \in b \Omega$, there exists a neighborhood $U$ of $p$ and a local defining function $r$ such that $-(-r)^{t}$ is plurisubharmonic on $U \cap \Omega$. Let $s=\frac{1+t}{2}$ and choose a neighborhood $U$ and defining function $r$ such that $-(-r)^{s}$ is plurisubharmonic on $U \cap \Omega$. Then, by Lemma 1.1:

$$
i \partial \bar{\partial}(-\log (-r)) \geq i s \frac{\partial r \wedge \bar{\partial} r}{r^{2}}
$$

Since $r$ and $\rho$ are both defining functions for $b \Omega$ on $U$, there must exist some constant $c>1$ such that $\frac{1}{c}<\frac{\rho}{r}<c$ on $U$. If we set $h=\log \left(\frac{c \rho}{r}\right)$, then we have $0<h<2 \log c$ on $U$, so we can define a new defining function:

$$
\rho_{p}=r\left(h+(s-t)^{-1}\right)^{s-t}
$$

For the sake of clarity, we initially assume that $\rho$ (and hence $\rho_{p}$ ) is $C^{2}$. The general case can then be obtained by approximation (see $[\mathrm{Ha}]$ ). Referring again to Lemma 1.1, we wish to prove that:

$$
i \partial \bar{\partial}\left(-\log \left(-\rho_{p}\right)\right) \geq C_{0} \phi\left(-\rho_{p}\right) \omega+i t \frac{\partial \rho_{p} \wedge \bar{\partial} \rho_{p}}{\rho_{p}^{2}}
$$


By construction, we have:

$$
i \partial \bar{\partial}\left(-\log \left(-\rho_{p}\right)\right)=i \partial \bar{\partial}(-\log (-r))-i(s-t) \partial \bar{\partial} \log \left(h+(s-t)^{-1}\right)
$$

To evaluate the second term, we check:

$$
\begin{aligned}
i \partial \bar{\partial} \log \left(h+(s-t)^{-1}\right) & =i \partial\left(\frac{\bar{\partial} h}{h+(s-t)^{-1}}\right) \\
& =i \frac{\partial \bar{\partial} h}{h+(s-t)^{-1}}-i \frac{\partial h \wedge \bar{\partial} h}{\left(h+(s-t)^{-1}\right)^{2}}
\end{aligned}
$$

Since:

$$
i \partial \bar{\partial} h=i \partial \bar{\partial}(-\log (-r))-i \partial \bar{\partial}(-\log (-\rho))
$$

we can combine (3.6), (3.7), and (3.8) to obtain:

$$
\begin{aligned}
& i \partial \bar{\partial}\left(-\log \left(-\rho_{p}\right)\right) \\
& =i\left(1-\frac{s-t}{h+(s-t)^{-1}}\right) \partial \bar{\partial}(-\log (-r))+i \frac{s-t}{h+(s-t)^{-1}} \partial \bar{\partial}(-\log (-\rho)) \\
& +i \frac{s-t}{\left(h+(s-t)^{-1}\right)^{2}} \partial h \wedge \bar{\partial} h
\end{aligned}
$$

We need $1-\frac{s-t}{h+(s-t)^{-1}}>0$ to proceed. Since $h>0$, we have:

$$
1-\frac{s-t}{h+(s-t)^{-1}}>1-(s-t)^{2}
$$

Note that $s-t=\frac{1-t}{2}$, so $0<s-t<\frac{1}{2}$ and hence $(s-t)^{2}<\frac{1}{4}<1$. Thus the needed inequality holds, and by substituting (3.3) and (3.10) we have, for some appropriate value of $C_{0}$ :

$$
\begin{aligned}
i \partial \bar{\partial}\left(-\log \left(-\rho_{p}\right)\right) & \geq C_{0} \phi\left(-\rho_{p}\right) \omega+i\left(1-(s-t)^{2}\right) s \frac{\partial r \wedge \bar{\partial} r}{r^{2}} \\
& +i \frac{s-t}{\left(h+(s-t)^{-1}\right)^{2}} \partial h \wedge \bar{\partial} h
\end{aligned}
$$

Here we may assume that $\phi(-\rho) \approx \phi\left(-\rho_{p}\right)$, since $\phi(x)$ is at worst $O\left(\frac{1}{x}\right)$ (in the strictly pseudoconvex case).

To handle the remaining terms, we observe that:

$$
\begin{aligned}
\frac{\partial \rho_{p}}{\rho_{p}} & =\partial\left(\log \left(-\rho_{p}\right)\right) \\
& =\partial(\log (-r))+(s-t) \partial \log \left(h+(s-t)^{-1}\right) \\
& =\frac{\partial r}{r}+\frac{(s-t) \partial h}{\left(h+(s-t)^{-1}\right)}
\end{aligned}
$$

so: 


$$
\begin{aligned}
i \frac{\partial \rho_{p} \wedge \bar{\partial} \rho_{p}}{\rho_{p}^{2}} & =i \frac{\partial r \wedge \bar{\partial} r}{r^{2}}+i \frac{(s-t)^{2} \partial h \wedge \bar{\partial} h}{\left(h+(s-t)^{-1}\right)^{2}} \\
& +i \frac{\partial r}{r} \wedge \frac{(s-t) \bar{\partial} h}{\left(h+(s-t)^{-1}\right)}+i \frac{(s-t) \partial h}{\left(h+(s-t)^{-1}\right)} \wedge \frac{\bar{\partial} r}{r}
\end{aligned}
$$

Using (3.13) to compare the last term in (3.5) with the last two terms in (3.11), we see that we need:

$$
\begin{aligned}
& i\left(\left(1-(s-t)^{2}\right) s-t\right) \frac{\partial r \wedge \bar{\partial} r}{r^{2}}+i(1-t(s-t))(s-t) \frac{\partial h \wedge \bar{\partial} h}{\left(h+(s-t)^{-1}\right)^{2}} \\
& -i t(s-t) \frac{\partial r}{r} \wedge \frac{\bar{\partial} h}{\left(h+(s-t)^{-1}\right)}-i t(s-t) \frac{\partial h}{\left(h+(s-t)^{-1}\right)} \wedge \frac{\bar{\partial} r}{r} \geq 0
\end{aligned}
$$

Checking the first coefficient, we have $\left(1-(s-t)^{2}\right) s-t=s-t-s(s-t)^{2}=$ $(s-t)\left(1-(s-t)^{2}\right)>0$. For the second coefficient, we know $0<s-t<\frac{1}{2}$, so $(1-t(s-t))(s-t)>\left(1-\frac{t}{2}\right)(s-t)>\frac{s-t}{2}>0$. Viewing (3.14) as a $2 \times 2$ hermitian matrix, we have shown that the diagonal elements are positive, so it remains to check the determinant. If we first factor $(s-t)$ from each term, we have a determinant of:

$$
\begin{aligned}
& (s-t)^{2}\left(\left(1-(s-t)^{2}\right)(1-t(s-t))-t^{2}\right) \\
& =(s-t)^{2}\left(1-t^{2}-t(s-t)-(s-t)^{2}+t(s-t)^{3}\right)
\end{aligned}
$$

Since $1-t=2(s-t)$ and $1+t=2 s, 1-t^{2}=4 s(s-t)$, so (3.15) becomes:

$$
\begin{aligned}
& (s-t)^{3}\left(4 s-t-(s-t)+t(s-t)^{2}\right) \\
& =(s-t)^{3}\left(3 s+t(s-t)^{2}\right)>0
\end{aligned}
$$

A $2 \times 2$ matrix with positive entries on the diagonal and a positive determinant is positive, hence (3.14) is positive and the right hand side of (3.11) is greater than the right hand side of (3.5). The lemma follows.

We note that if $\phi(x)=x^{-2 \epsilon}$ (the subelliptic case), then this lemma gives us a defining function satisfying:

$$
i \partial \bar{\partial}\left(-\left(-\rho_{p}\right)^{t}\right) \geq C\left(-\rho_{p}\right)^{t-2 \epsilon} \omega
$$

With the special defining function give by Lemma 3.1, we are now ready to prove the main result of this section.

THEOREM 3.2. Let $M$ be a complex Kähler manifold with nonnegative sectional curvature. Let $\Omega \subset \subset M$ be a pseudoconvex domain with $C^{2}$ smooth boundary $b \Omega$. Let $\rho$ be a defining function for $\Omega$ satisfying (3.1) in $\Omega$. Then for every $0 \leq s<\frac{1}{2}$ the $\bar{\partial}$-Neumann operator $N$ is compact in $W_{(p, q)}^{s}(\Omega)$ for all $0 \leq p \leq n, 1 \leq q \leq n$.

Proof. Cover $b \Omega$ with some finite collection of open sets $U_{p}$ such that for each $U_{p}$ there is a defining function $\rho_{p}$ satisfying the conclusions of Lemma 3.1 for $t=2 s$. We may assume that the value of the constant $C$ is uniform with respect to $p$. Let $\epsilon>0$ be given, and choose any neighborhood $V$ of $b \Omega$ such that $C \phi\left(-\rho_{p}(z)\right)>\frac{1}{\epsilon}$ on $U_{p} \cap V$ for any $p$. Let $\chi_{p} \in C_{0}^{\infty}(M)$ be a partition of unity with respect to the 
covering $\left\{U_{p}\right\} \cup\{\Omega\}$, and choose $\gamma \in C_{0}^{\infty}(V)$ such that $\gamma \equiv 1$ in a neighborhood of $b \Omega$. Then, for any $(p, q)$-form $g \in \operatorname{Dom}(\bar{\partial}) \cap \operatorname{Dom}\left(\bar{\partial}^{*}\right)$ with $q \geq 1$ on $\Omega$, we may apply the Bochner-Hörmander-Kohn-Morrey formula as in the proof of Theorem 2.2 to obtain:

$$
\left\|\bar{\partial}\left(\gamma \chi_{p} g\right)\right\|_{(s)}^{2}+\left\|\bar{\partial}^{*}\left(\gamma \chi_{p} g\right)\right\|_{(s)}^{2} \geq \frac{1}{\epsilon}\left\|\gamma \chi_{p} g\right\|_{(s)}^{2}
$$

for all $p$. Since the $\chi_{p}$ are independent of $\epsilon$, there is some constant $c_{0}$ independent of $\epsilon$ such that:

$$
\left\|\chi_{p} \bar{\partial}(\gamma g)\right\|_{(s)}^{2}+\left\|\chi_{p} \bar{\partial}^{*}(\gamma g)\right\|_{(s)}^{2}+c_{0}\|\gamma g\|_{(s)}^{2} \geq \frac{1}{\epsilon}\left\|\chi_{p} \gamma g\right\|_{(s)}^{2}
$$

Summing over $p$, we can find some constants $c_{1}$ and $c_{2}$ independent of $\epsilon$ such that:

$$
c_{1}\left(\|\bar{\partial}(\gamma g)\|_{(s)}^{2}+\left\|\bar{\partial}^{*}(\gamma g)\right\|_{(s)}^{2}\right)+c_{2}\|\gamma g\|_{(s)}^{2} \geq \frac{1}{\epsilon}\|\gamma g\|_{(s)}^{2}
$$

Since all constants are independent of $\epsilon$, we may replace $\epsilon$ with $\tilde{\epsilon}$ and set $\frac{1}{\epsilon}=$ $\left(\frac{1}{\tilde{\epsilon}}-c_{2}\right) \frac{1}{c_{1}}$ to obtain:

$$
\epsilon\left(\|\bar{\partial}(\gamma g)\|_{(s)}^{2}+\left\|\bar{\partial}^{*}(\gamma g)\right\|_{(s)}^{2}\right) \geq\|\gamma g\|_{(s)}^{2}
$$

In the interior we have Gårding's inequality, so for any $\epsilon>0$ we can always find $c_{\epsilon}>0$ such that:

$$
\epsilon\left(\|\bar{\partial}((1-\gamma) g)\|_{(s)}^{2}+\left\|\bar{\partial}^{*}((1-\gamma) g)\right\|_{(s)}^{2}\right)+c_{\epsilon}\|(1-\gamma) g\|_{-1}^{2} \geq\|(1-\gamma) g\|_{(s)}^{2}
$$

Hence, we can combine (3.17) and (3.18), adjust $c_{\epsilon}$ accordingly, and obtain:

$$
\epsilon\left(\|\bar{\partial} g\|_{(s)}^{2}+\left\|\bar{\partial}^{*} g\right\|_{(s)}^{2}\right)+c_{\epsilon}\|g\|_{-1}^{2} \geq\|g\|_{(s)}^{2}
$$

Such estimates are equivalent to compactness for the $\bar{\partial}$-Neumann operator (see $[\mathrm{KN}]$ or $[\mathrm{FS}]$ ). As usual, we can pass from weighted estimates to $W^{-s}$ estimates since this is an elliptic system. We have the dual estimates in $W^{s}$ as well, and can obtain the $L^{2}$ case by interpolation.

The subelliptic case is similar, and we omit the proof.

THEOREM 3.3. Let $M$ be a complex Kähler manifold with nonnegative sectional curvature. Let $\Omega \subset \subset M$ be a pseudoconvex domain with $C^{2}$ smooth boundary $b \Omega$. Let $\rho$ be a defining function for $\Omega$ satisfying (3.1) in $\Omega$ for $\phi(x)=x^{-2 \epsilon}$, where $0<\epsilon<\frac{1}{2}$. Then for every $0 \leq s<\frac{1}{2}-\epsilon$, the $\bar{\partial}$-Neumann operator $N$ is bounded from $W_{(p, q)}^{s-\epsilon}(\Omega)$ to $W_{(p, q)}^{s+\epsilon}(\Omega)$ for all $0 \leq p \leq n, 1 \leq q \leq n$.

We remark that in the Lipschitz case, the conclusions of Theorems 3.1 and 3.2 still hold for $s=0$ (see [Ha]). However, it is unknown whether such results are possible when $s>0$, even for arbitrarily small $s$. 


\section{REFERENCES}

[Ba] Barrett, D. E., Behavior of the Bergman projection on the Diederich-Fornaess worm, Acta Math, 168 (1992), pp. 1-8.

[BC] Berndtsson, B and Charpentier, P., A Sobolev mapping property of the Bergman kernel, Math. Zeitschrift, 235 (2000), pp. 1-10.

[BS] Boas, H. P. and Straube, E. J., Sobolev estimates for the $\bar{\partial}$-Neumann operator on domains in $\mathbf{C}^{n}$ admitting a defining function that is plurisubharmonic on the boundary, Math. Zeit., 206 (1991), pp. 81-88.

[Ca] Catuin, D., Subelliptic estimates for the $\bar{\partial}-$ Neumann problem on pseudoconvex domains, Ann. Math.,, 126 (1987), pp. 131-191.

[CS] Chen, S.-C. And Shaw, M.-C., Partial Differential Equations in Several Complex Variables, American Math. Society-International Press, Studies in Advanced Mathematics, Volume 19, Providence, R.I., 2001.

[CS1] CaO, J. And Shaw, M.-C., A new proof of the Takeuchi theorem, Proceedings of the Conference "CR Geometry and PDEs" Trento, Italy, Interdisplinare di Mathematica, 4 (2005), pp. $65-72$.

[CS2] CaO, J. And Shaw, M.-C., The $\bar{\partial}$-Cauchy problem and nonexistence of Lipschitz Levi-flat hypersurfaces in $\mathbb{C} P^{n}$ with $n \geq 3$, Math. Zeit., 256 (2007), pp. 175-192.

[CSW] CAO, J., ShaW, M.-C. AND Wang L., Estimates for the $\bar{\partial}$-Neumann problem and nonexistence of Levi-flat hypersurfaces in $\mathbb{C} P^{n}$, Math. Zeit, 248 (2004), pp. 183-221; Erratum, pp. 223-225.

[DA] D'Angelo, J., Real hypersurfaces, order of contact, and applications, Ann. Math.,, 115 (1982), pp. 615-637.

[De] Demailly, J.-P., Mesures de Monge-Ampère et mesures pluriharmoniques, Math. Zeit., 194 (1987), pp. 519-564.

[DF] Diederich, K. And Fornaess, J. E., Pseudoconvex domains: Bounded strictly plurisubharmonic exhaustion functions, Invent. Math.,, 39 (1977), pp. 129-141.

[FK] Folland, G. B. And Kohn, J. J., The Neumann Problem for the Cauchy-Riemann Complex, Ann. Math. Studies 75, Princeton University Press, Princeton, N.J., 1972.

[FS] Fu, S. and Straube, E. J., Compactness in the $\bar{\partial}$-Neumann problem. In Complex Analysis and Geometry, Proceedings of a Conference at Ohio State University 9, Walter De Gruyter, Berlin, 2001, pp. 141-160.

[GW] Greene, R. E. And Wu, H., Function Theory on Manifolds Which Possess a Pole, SpringerVerlag, Berlin, Heidelberg, New York, 1979.

[Ha] Harrington, P. S., A quantitative analysis of Oka's lemma, Math. Zeit, 256 (2007), pp. 113138.

[HI] Henkin, G. M. And Iordan, A., Regularity of $\bar{\partial}$ on pseudoconcave compacts and applications, Erratum: Asian J. Math., vol 7, (2003) No. 1, pp. 147-148), Asian J. Math., 4 (2000), pp. 855-884.

[Hö1] Hörmander, L., An Introduction to Complex Analysis in Several Complex Variables, Third Edition, Van Nostrand, Princeton, N.J., 1990.

[Hö2] Hörmander, L., $L^{2}$ estimates and existence theorems for the $\bar{\partial}$ operator, Acta Math., 113 (1965), pp. 89-152.

[KeR] Kerzman, N. And Rosay, J.-P., Fonctions Plurisousharmoniques d'exhaustion bornées et domaines taut, Math. Ann., 257 (1981), pp. 171-184.

[Ko 1] Koнn, J. J., Subellipticity of the $\bar{\partial}$-Neumann problem on pseudoconvex domains: sufficient conditions, Acta Math.,, 142 (1979), pp. 79-122.

[Ko 2] Konn, J. J., Quantitative estimates for global regularity. Analysis and geometry in several complex variables (Katata, 1997), Trends Math., Birkh" user Boston, Boston, MA (1999), pp. $97-128$.

[KoN] Kohn, J. J., And Nirenberg, L., Noncoercive boundary value problems, Comm. Pure Appl. Math.,, 18 (1965), pp. 443-492.

[OS] Ohsawa, T. and Sibony, N., Bounded P.S.H Functions and Pseudoconvexity in Kähler Manifolds, Nagoya Math. J., 149 (1998), pp. 1-8.

[Si] SiU, Y.-T., Nonexistence of smooth Levi-flat hypersurfaces in complex projective spaces of dimension $\geq 3$, Ann. Math., 151 (2000), pp. 1217-1243. 
[Ta] Takeuchi A., Domaines pseudoconvexes infinis et la métrique riemannienne dans un espace projectif, J. Math. Soc. Japan, 16 (1964), pp. 159-181. 
